Review

\title{
Biomedical Nanoparticles: Overview of Their Surface Immune-Compatibility
}

\section{Olimpia Gamucci, Alice Bertero, Mariacristina Gagliardi and Giuseppe Bardi *}

Center for MicroBioRobotics @SSSA, Istituto Italiano di Tecnologia, Viale Rinaldo Piaggio 34, 56025 Pontedera, Italy; E-Mails: olimpia.gamucci@iit.it (O.G.); alice.bertero@iit.it (A.B.); mariacristina.gagliardi@iit.it (M.G.)

* Author to whom correspondence should be addressed; E-Mail: giuseppe.bardi@iit.it;

Tel.: +39-050-883-028; Fax: +39-050-883-101.

Received: 11 October 2013; in revised form: 8 January 2014 / Accepted: 30 January 2014 /

Published: 12 February 2014

\begin{abstract}
Diagnostic- and therapeutic release-aimed nanoparticles require the highest degree of biocompatibility. Some physical and chemical characteristics of such nanomaterials are often at odds with this requirement. For instance, metals with specific features used as contrast agents in magnetic resonance imaging need particular coatings to improve their blood solubility and increase their biocompatibility. Other examples come from the development of nanocarriers exploiting the different characteristics of two or more materials, i.e., the ability to encapsulate a certain drug by one core-material and the targeting capability of a different coating surface. Furthermore, all these "human-non-self" modifications necessitate proofs of compatibility with the immune system to avoid inflammatory reactions and resultant adverse effects for the patient. In the present review we discuss the molecular interactions and responses of the immune system to the principal nanoparticle surface modifications used in nanomedicine.
\end{abstract}

Keywords: immune system; nanomaterials; immunogenicity; immunotoxicity; biodistribution; mononuclear phagocytic cells; surface modifications 


\section{Introduction}

The focus of the present review is to describe the main molecular mechanisms of host defense towards the principal surface modifications of nanoparticles (NPs) used in medical fields as diagnostics or therapeutics.

A complex variety of organic materials (e.g., polymers, dendrimers, liposomes, proteins and carbohydrates), allotropic forms of carbon (e.g., fullerenes, carbon nanotubes) and inorganic nanosized particles (e.g., quantum dots, silica, gold or iron core NPs) are currently under investigation for drug delivery and imaging purposes $[1,2]$.

The nano size of drug delivery vectors is designed to improve solubility, pharmacokinetics and biodistribution of small drugs, vaccines or other active molecules, otherwise less effective. Together with these advantages, the introduction of such nano-systems may pose biocompatibility concerns that go beyond the drug toxicological profile.

Complex living organisms have perfected their defense mechanisms towards exogenous pathogens throughout the course of evolution. The immune system is able to distinguish between self and non-self substances. The foreign molecules able to elicit immune responses by binding to specific host receptors are called antigens (antibody generators). The immune system ensures a plethora of selective response against several non-self molecules retaining immunological memory and preventing injury to host cells during responses to microbes. Recognition and subsequent clearance of these alien elements are important physiological mechanisms employed by the immune system to maintain the body homeostasis [3].

\section{A Brief Description of Immune System Main Features}

Different and subsequent lines of defense of increasing specificity are deployed by the immune system to eradicate infections by exogenous organisms. Physical barriers, like the skin and mucosa, are the first protection of the innate immunity to prevent the entrance of pathogens into the body. If infective microorganisms overcome these barriers, another line of innate defense provides an immediate but non-specific response. This line of control is carried out by different cellular and biochemical mediators. Phagocytic cells, like neutrophils or macrophages, play a major role in wiping out the foreign pathogen by encapsulating and destroying it with specific enzymatic reaction cascades. These cells recognize unique molecular structures of microbial pathogens, called Pathogen-Associated Molecular Patterns (PAMPs), by means of specific pattern-recognition receptors. Well known examples of PAMP-receptors are the Toll-like receptors (TLRs) which recognize various microbial molecules including the Gram-negative bacteria wall component lipopolysaccharide (LPS), the lipoteichoic acid (present on the wall of Gram-positive bacteria) receptor or the receptors for mannose-rich oligosaccharides present on the fungi. Innate immune cells are also able to respond to secondary effects like cell damages caused by infections. Damage-Associated Molecular Patterns (DAMPs) indicate cell injuries induced by a myriad of reasons, including toxins, traumas or decreased nutrient supply. Stress induced proteins, nuclear proteins or crystal (i.e., monosodium urate) are recognized by DAMP receptors that initiate immune responses. Furthermore, blood proteins can directly interact with the undesired invader and regulate its fate. A heterogeneous group of cell derived proteins, named cytokines, synchronize all these immune response mechanisms [3]. 
By means of evolution, most of the vertebrates also possess a further level of protection, the adaptive immune response, which can be activated by the innate immunity if pathogens have not been successfully eliminated. The adaptive immune system improves its response during an infection and "memorizes" pathogen features once it has been eliminated. The immunological memory allows a faster and stronger reaction by subsequent exposure to an already recognized element. Indeed, this mechanism is the basic principle of vaccination. The adaptive immunity is also called "acquired immunity", as a consequence of its responses against non-self elements "acquired" by experience. The peculiar cells of adaptive immunity are the lymphocytes. These antigen-specific cells display receptors that are able to recognize an incredible number of exogenous pathogens and to distinguish between very closely related molecular structures.

We can describe two different types of adaptive responses, named humoral and cell-mediated immunity, often working contemporaneously and in conjunction with the innate system. The humoral immunity is mediated by proteins called antibodies. This family of proteins is secreted by B lymphocytes (or B cells) once differentiated into antibody-secreting plasmacells. The process of recognition of pathogens by B lymphocytes is also mediated by antibody receptors expressed on the cell surface that trigger an intracellular signaling leading to cell differentiation upon foreign molecule binding. In contrast, cell-mediated reactions begin with a coordinated interaction between a different type of lymphocytes, the T lymphocytes (or T cells), and Antigen Presenting Cells (APCs). T cells are not capable of producing antibodies and have a restricted specificity for antigens [4]. They recognize only foreign peptides bound to host molecules, the major histocompatibility complex (MHC) exposed on the presenting cells. One of the main tasks of adaptive cell-mediated immunity is to destroy microbes that survive and proliferate inside the cells, like viruses. Functionally different subpopulations of $\mathrm{T}$ lymphocytes play a role in the various kinds of infections. All these subsets are grouped in two main families, known as T helper (THLs) and cytotoxic T cells (CTLs). The antigenic stimulation induces $\mathrm{T}$ helper cells to secrete cytokines, which in turn foster proliferation and differentiation of $\mathrm{T}$ cells, B cells, macrophages and other leukocytes. Cytotoxic T lymphocytes are equipped with specific set of reactive proteins capable to kill cells that expose foreign antigens on their surface. A third group of lymphocytes is represented by natural killer (NK) cells, which are principally involved in innate response towards microbes and pathogens [5]. Lymphocytes and APCs circulate through the body and home to the target sites of antigen exposure. Lymphoid organs, like spleen, liver or lymph nodes represent the anatomical districts where these cells increase the possibility of antigen presentation and start an immune response [6].

All the immune cells travel within the body in a strictly regulated manner. Differentiated cells from all the tissues provide signals to drive the immune cell travelling, mainly releasing chemokines (chemoattractant cytokines) [7]. This very special type of polypeptides has a conserved structure which is maintained in the evolution, highlighting the importance of their role in cell migration. The tissue modifications happening during inflammatory processes are intended for leukocytes recruitment from the circulation to the injured tissue with the purpose of destroying pathogens. Indeed, the majority of the cell movements implicated in the physiological tissue rearrangements and pathological conditions rely on chemokine driven signaling [8]. 
As well as microbes, most of the engineered nanomaterials that enter the body are recognized as non-self by the immune system, which efficiently detects and tries to eliminate them through the described mechanisms [9]. This biological event makes the immune system a crucial obstacle to deal with, in order to apply nanotechnology to biomedicine.

\section{Nanoparticle Interaction with Blood Components and Uptake by Phagocytic Cells}

Critical physical and chemical features of NPs regulate the interaction with the immune system. Surface electrostatic charges of engineered nanomaterials are fundamental to define immune responses [10]. Positive-charged surfaces on the particles are often more cytotoxic than anionic and neutral ones. Cationic NPs bind more efficiently than anionic charged or neutral molecules to the negatively charged plasma membrane of target cells, which expose the negative charges of the phospholipidic groups. Once NPs have entered the cell, NP surface positive charge slows down the acidification of endosomes (responsible for the transport pathway from the plasma membrane to the lysosome), thereby delaying the endosome-lysosome transition. Moreover, they can cause more pronounced disruption of plasma-membrane integrity, stronger mitochondrial, lysosomal damage, and increased number of autophagosomes [11,12]. Positively charged elements introduced into cells may also form complexes with the negatively charged nucleic acids raising genotoxicity concerns. However, a net positive surface charge helps the binding to plasma membranes. This physical effect can be beneficial for drug delivery particles.

The different uptake preferences of phagocytic and nonphagocytic cells for cationic and anionic NPs are important for the efficacy and selectivity of NPs. In general, neutral particles show lower interaction with the cell membrane than charged (cationic or anionic) nanoparticles of the same size, due to the lower number of electrostatic interactions between NP-surface and charged cell membranes. Designing optimal coatings for drug delivery carriers requires a precise characterization of these physical interactions.

Drug or gene delivery nanocarriers for systemic injection in the bloodstream come across a biological milieu composed of cells, proteins and solutes [13]. Injected NPs almost instantaneously adsorb and bind different proteins, whose affinity kinetics depends on the nano-material surface, charge and size. The protein layer created on the particle surface is called "corona" and it influences particle biodistribution and circulation time. Proteins that increase the clearance of exogenous particles by specialized mononuclear phagocytes are called opsonins. Blood released antibodies, for example, principally work as opsonins enhancing phagocytosis. Molecules that activate the complement system (see below) are also considered opsonins. Their binding to NPs injected in the bloodstream leads to the attachment of macrophages followed by cell internalization. This type of internalization is highly regulated by cells surface receptors and their capability of binding to the particular protein corona attached to the particle. A detailed characterization of NP-specific opsonization process will help to design nanoparticle with future clinical relevance.

The protein corona hiding the surface of NPs determines the effective size and the final beneficial/dangerous effects of the material. Besides enhancing particle recognition by the host immune cells, protein adsorption/opsonization increases the hydrodynamic diameter (HD) which contributes to modify NP accumulation and tissue distribution. Generally HD is greatly larger than the NP diameter measured after their synthesis. The HD of particles injected in the bloodstream is inversely related to 
glomerular filtration rate in the kidney, ultimately regulating the blood half-life of the solutes [14]. Molecules with an HD $<5 \mathrm{~nm}$ achieve a rapid equilibrium with the extravascular extracellular space (EES), whereas larger particles have prolonged circulatory times due to slow transport across the endothelium $[15,16]$.

The opsonization process of NPs may involve the blood proteins of the complement system, which refers to about 30 small blood molecules (serum proteins and cell membrane receptors) synthesized by the liver [17]. This system helps and "complements" the action of innate and adaptive immunity and has an important role in leukocytes chemotaxis and bacteria lysis. Upon stimulation, specific proteases cleave downstream peptides to release cytokines, thus initiating an amplifying the proteolytic cascade to further cleavages. The end-result is a massive amplification of the response and subsequent activation of APCs, T and B cells. Complement can be activated through three different biochemical ways: the classical, the alternative and the lectin pathway. The several types of NPs can differently activate the complement system through one specific pathway or a combination of them [18]. The specificity of the activated pathway by NPs depends on their surface properties, such as the conformation of a particular polymer coating [19-21].

The activation of complement protein cascades can be responsible for some adverse effects (i.e., hypersensitivity and anaphylaxis). To avoid such events, nano-formulations intended for systemic administration of drug carriers are usually designed to prevent the complement activation.

As previously mentioned, NP physicochemical properties regulate their recognition and uptake by phagocytic cells. In the past decades it was believed that phagocytosis was restricted to large matter $(>1 \mu \mathrm{m})$. However, like other cells, macrophages can also use different routes of internalization like endocytosis and macropinocytosis to uptake nanosized particles. It has been observed that nanoparticles $>500 \mathrm{~nm}$ are primarily and more efficiently internalized via macropinocytosis or phagocytosis mechanism than smaller ones with the same composition and surface properties [18,22]. NPs with dissimilar morphologies interact with macrophages in a different way. As expected, the longest dimensions exhibit the strongest attachment with macrophages membranes. Nonetheless, virus-sized particles (20-200 nm) are efficiently taken up by macrophages or APCs, but mainly via clathrin-coated mediated endocytosis [23].

Once internalized within immune cells, NPs can activate cytoplasmic multiprotein complexes called inflammasomes, which are involved in the initiation of inflammatory responses [24]. These complexes induce the proteolysis of inflammatory cytokine precursors and the following release of the bioactive mediators. Endo/lysosome compartment damage and the release of hydrolytic enzymes, such as Cathepsin B seem to play a major role in NP-induced inflammasome-dependent interleukin-1 $\beta$ (IL-1 $\beta$ ) release by macrophages and DCs [25].

Unfortunately, the information regarding the interaction of nanomaterials with the immune system is still limited and fragmentary. Many results have been obtained in vitro using mouse or human immortalized cell lines. The differences between mouse and human immune systems rise up some concern on the potential clinic reliability. A second issue to be considered is the constitutive release of inflammatory factors or growing hormones by immune cell lines, otherwise suppressed by primary cells or temporary expressed after external stimuli. Furthermore, the several synthesis processes to produce the same nanomaterials may introduce discrepancies in the resulting in vivo outcomes. 
In this review we report and comment on what is known in literature on the immunological characterization of the principal inorganic and organic materials usually exploited in NP coating for biomedical applications. To better focus on the surface material-related immune events, we will not describe and discuss NP functionalization with peptides or other biological moieties intended for specific cell targeting.

\section{NP Surface Coatings}

The employment of synthetic polymers to coat NPs increases their stability and solubility with advantageous effects on cytotoxicity or inflammatory responses following administration [26]. Active molecules can be encapsulated (adsorbed or bound) into the coated carriers to protected them from metabolizing enzymes. Specific NP surfaces can confer protection to non-target tissues from a possible unspecific toxic action of the drug payloads leading to side effects of the pharmaceutics. On the other hand, they protect the drug from premature release or degradation in the biological environment of the body, preserving their efficacy.

As well as delivery carriers, different type of shells cover NPs aimed at imaging and more in general at diagnosis. For instance, contrast agents for Magnetic Resonance Imaging (MRI), like super-paramagnetic iron oxide (SPIO) NPs or paramagnetic gadolinium-labeled NPs are often coated with polymers to increase their solubility and biocompatibility [27].

Conversely, the coating can be chosen to produce immune-stimulant reactions, as demonstrated by the application of NPs as adjuvant in vaccines production, which is becoming a field of fast development in pharmaceutical industry [28]. In this case, reactive surfaces stimulate specific immune cells in order to increase the response to antigens.

Therefore, NP surface features and materials must be considered as key factors that may significantly contribute to adverse immune reactions and toxicity in current healthcare practice. In the following paragraphs, representative observations will describe the type of interaction that the mainly used NP-coating materials have with the immune system and their proved or potential effects.

\section{Synthetic Polymeric Coatings}

\subsection{Polyethylene Glycol (PEG)}

Hydrophobic surfaces generally tend to exclude water molecules causing aggregation and precipitation of unstable colloids with limited biological applications. NP surface can be modified by using highly hydrophilic polymers, such as poly(ethyleneglycol) (PEG) [26]. PEG is a polyether chain able to absorb water and generate hydrogen bonds that allow solubilization in polar solvents and the stabilization of the colloid either in acidic or basic $\mathrm{pH}$ environments. As we mentioned before, charged nanoparticles bind more proteins than particles with neutral surfaces. Surface neutralization by coating with PEG is one of the best approaches to protect nanoparticle surface from protein binding.

Two different approaches are mainly used to obtain PEG-coated nanoparticles: PEG adsorption or the covalent attachment of the polyether chain on the surface, usually called PEGylation [29]. PEG adsorption is the simpler approach, but it is barely useful in the case of NPs released in a biological environment. Since this polymer is highly water-soluble, the coating might be immediately dissolved in 
salty fluids. PEGylation represents the best way to stably attach the polyether chain on the surface for biomedical applications. A third way to obtain PEGylation is using amphiphilic di-block copolymers to form NPs. In this case, self-assembling micellar structures can be produced and loaded with the desired cargo [30].

As consequence of PEG coating NP internalization by phagocytes is reduced prolonging their circulation time when released in the blood [13]. However, PEG covering of some materials like liposomes does not protect from complement activation. The presence of specific chemical groups on the polymer shell may modulate this effect. For instance, linkers like hydroxy and thiol-groups do not activate the complement cascade, while methoxy groups potently trigger the cleavage reaction [21]. Different structural conformation and chemical group charges may determine the complement binding with associated immune responses.

Most of the data present in literature describing antigenic responses to NPs surface coatings are provided by experiments using PEGylated liposomes. Repeated systemic administrations of these nano-systems induce an antibody-dependent rapid clearance of the second dose from the circulation [13]. This phenomenon is called accelerated blood clearance (ABC). The $A B C$ effect seems to be accompanied by the accumulation of both PEGylated and uncoated liposomes in the liver and spleen immune resident cells. Phagocytic cells depletion attenuates this process, suggesting that macrophages are involved in NPs accelerated clearance. The rate of the ABC phenomenon depends on many factors, like the dose, the interval between the administered doses, the NP size, the surface charge, the liposomal composition and the PEG density.

Nowadays, PEG coatings show many advantages to produce "stealth" NPs able to avoid a major activation of immune system. This is proved by its abundant use as sole coating or in combination with other polymers. Nevertheless, clinical studies employing PEGylated NPs for long time are not available to assure its complete immune-safety.

\subsection{PLGA}

Poly(lactic-co-glycolic acid) (PLGA) is one of the most successfully developed polymers for therapeutic devices, owing to its biodegradability and biocompatibility [31]. PLGA is synthesized by co-polymerization of two different monomers, the cyclic dimers of glycolic acid and lactic acid. Depending on the ratio of lactide to glycolide used for the polymerization, different forms of PLGA can be obtained. Although the homopolymers of lactic acid (polylactide) and glycolic acid (polyglycolide) show poor solubility, PLGA can be dissolved by a wide range of common solvents.

Under normal physiological conditions, PLGA undergoes hydrolysis in the body and produces its original monomers. Since they are by-products of various metabolic pathways in the body, there is minimal systemic toxicity associated with the use of PLGA for drug delivery or other biomedical applications.

PLGA is described in the literature as a promising carrier adjuvant for nasal subunit vaccines. Glycol chitosan coated PLGA (GC-PLGA) NPs were found to elicit relatively stronger immune response as compared to chitosan coated PLGA (C-PLGA) and PLGA NPs after nasal administration [32]. GC-PLGA NPs showed a better mucoadhesivity, with consequently prolonged nasal residence time. Furthermore, GC-PLGA NPs resulted in a more efficient antigen uptake and transport across the nasal 
mucosa and into the circulatory system. The coating of NPs with chitosan and glycol chitosan did not affect the integrity and release profile of the antigen.

Surface-modified PLGA microspheres (cationic PLGA microspheres) were also described as potent nasal delivery systems for vaccines where mucosal, humoral and cellular responses after bacterial and viral pathogens invasion are required [33].

It is worth noting that PLGA particulate vaccine adjuvants enhance LPS-induced NALP3 inflammasome and Caspase 1 activation leading to increased IL-1 $\beta$ secretion [34,35]. This observation emphasizes the complexity of immune responses to NPs even for those usually considered non-toxic and biodegradable. It can be hypothesized that the presence of PLGA coating helped to increase the efficiency of the adjuvant delivery without a direct involvement in the immune response. The enhancement of an inflammatory pathway born after particle internalization, that is, the inflammasome activation, would suggest the hydrolyzation of the PLGA coating within the cell.

The advantage of using PLGA as a good and biodegradable protection for the desired cargo also seems a good choice for the immune-compatibility of the polymer, based on the absence of detrimental effects in the available literature.

\subsection{Dendrimers}

Dendrimers molecules are highly-branched symmetric structures which are synthesized in a layer-by-layer fashion expressed in "generations" (G) around a core unit. This structure has repeated dendrons with a single chemical linking group, called focal point. Their synthesis results in high level of control over size, branching points and surface functionality. Several kinds of inorganic nanoparticles, coated with dendrimers are reported in literature for drug and gene delivery [36-38] or imaging [39].

Many detailed results are available on dendrimer toxicity either in vitro or in vivo [40]. However, information on the immune system reactions to dendrimer is still less well explored. Glycodendrimers containing various surface modifications have been designed as anti-infective ligands with anti-viral or antimicrobial effects. As examples, a polysulfonate G4 polyamidoamine (PAMAM) dendrimer show the ability to block HIV-1 and HIV-2 activity in vitro [41]. Although the mechanism of infection prevention is mainly related to structural entrapment of the virus by the branched structure, dendrimer-related antimicrobial activity may also involve immune-modulation. For instance, $N$-acetyl-glucosamine-coated G1 PAMAMs administered in mice bearing subcutaneous melanoma model, decrease tumor growth and increase mice survival. These results were accompanied by an increase of CD69+ cells in the spleen and the tumor tissue, associated to the up-regulation of IL-1h, IFN-g, TNF-a and IL-2 [42]. Another indication of such phenomenon is provided by the over-expression of pro-inflammatory chemokines and cytokines, namely MIP-1a, MIP-1h, IL-8, TNF-a, IL-1h and IL-6 induced in human dendritic cells and macrophages by glucosamine-modified G3.5 PAMAMs [43].

Many different dendrimer compositions can be synthesized and each one should be studied using primary immune cells and animal models in order to understand and eventually classify the different features of immune responses. It is clear that cationic dendrimers can damage cell membranes and this event can activate innate inflammatory reactions mediated by DAMP receptors, which could recognize cell components escaping from their intracellular location. 


\section{Natural Polymeric Coatings}

Among the natural polymers, chitosan, sodium alginate and dextran are frequently used to cover NPs. However, the information on immune effects is often restricted to the bulk material or to some specific application.

\subsection{Chitosan}

Chitosan (Chi) is a linear polysaccharide, extensively diffused in biomedical applications for its excellent properties including rapid blood clotting, biodegradability, non-toxicity, high charge density, gelling and mucoadhesivity. These features give to chitosan an immense potential for various pharmaceutical applications, such as the coating or entrapment of biochemicals, drugs and antigenic molecules. An example is represented by chitosan microspheres, which have been studied as promising carrier systems for mucosal vaccination (especially oral and nasal) to induce enhanced immune responses [32].

This natural polymer can be employed both for inorganic and organic nano-structures. Literature reports chitosan coatings of different metallic NPs [44,45]. Chi has been shown to enhance the function of immune cells, such as neutrophils and macrophages stimulating the production of cytokines and growth factors [46-49]. However, Chi is also controversially employed as a "fat binder" to reduce body weight. The mechanism of interaction between chitosan and fat is not well understood and has not been proved clinically. No indications of specific immune responses have been clearly shown following oral administration of chitosan tablets or pills. On the other hand, due to its adjuvant properties that increase inflammatory responses, caution should be used before choosing this polysaccharide as NP coating.

\subsection{Sodium Alginate}

Sodium alginate is a hydrophilic, water soluble and biocompatible polysaccharide obtained by marine brown algae. Alginate consists of $\alpha$-L-guluronate and $\beta$-D-mannuronate, arranged in a block structure as homopolymer (polyguluronate or polymannuronate) or heteropolymer (a mixed sequence of these residues).

It is often employed as a coating for magnetic [50] and polymeric NPs [51,52]; alginate oligomers obtained by enzymatic digestion of alginate polymer are able to induce the secretion of high levels of inflammatory cytokine and chemokines, like MCP-1, RANTES in vitro and in vivo [53,54]. Combinations of alginate and poly-lactide-co-glycolide acid (PLGA) are developed for vaccination purposes. Immunization studies in Balb/c mice by intradermal route demonstrated that incorporation of alginate elicited humoral and cellular immune responses [33,55].

Enhancement of non-specific immune responses demonstrated in vivo suggests the employment of alginate as coating for NPs aimed at immune stimulation. Either for vaccine adjuvant properties or for diagnostic purposes to reveal deficient innate immune reaction, this brown algae-derived anionic polysaccharide may represent a useful tool in biomedicine applications of NPs. 


\subsection{Dextran}

Dextran is a complex, branched polysaccharide composed of many glucose molecules. It is widely used in medicine as an anti-thrombotic (anti-platelet action) and blood viscosity reducer. Larger dextrans, which do not cross the vessels, are potent osmotic agents, and thus volume expanders in hypovolemia.

Dextran is also diffused for the realization of metallic NP coatings to protect core oxidation. Dextran coatings can be obtained by adsorption [56], chemical functionalization [27,57] or directly during nanoparticle formation [58].

Even if extensively employed for NP shells, a few studies investigated the effects of such surfaces on the immune system. Potential immune stimulatory effects on mouse splenocytes have been achieved using drug loaded chitosan-carboxymethyl dextran NPs (CDNP) [59]. Unfortunately, it is difficult to understand the specific contribution or role of the dextran in those kinds of immune responses. The complex of two or more polymers can create surfaces with very different characteristics and immune reactions.

Immune effects resulting from the release of free dextran molecules (not in nano-complexes) are not useful to establish its immunogenicity as NP coating. Many inflammatory reactions depend on the molecular weight of the chosen polysaccharide, its branching structure and the administered dose.

\subsection{Starch}

Starch is a different natural polymer produced by plants such as corn, potato, rise and cassava. It is composed of two biomolecules, namely amylose and amylopectin. Amylose is a linear polymer of glucose units mainly linked with alpha-1,4-bonds. Amylopectin, an extremely high molecular weight polymer, has the same backbone structure of amylose, but with many alpha-1,6-linked branch points. This polymer is highly biocompatible and biodegradable, with different physicochemical properties according to the type of starch source [60].

Due to all these features, starch is widely used in many different biomedical applications ranging from skin topical release [61] to degradable drug microsphere carrier [62]. Starch based coating have also been employed for gold [63] or iron-core magnetic NPs [64,65].

Although commonly used, the literature regarding immune system interaction with starch coated NPs is poor. It is unclear whether stereotyped immune reactions can be associated with the starch surface. Due to its wide presence in food and its biodegradability, starch is considered a "safe" material. It is expected, however, that potential allergic reaction will be experienced only in a restricted number of sensitive human recipients, as well as for the other materials.

\section{Inorganic NP Coatings}

Inorganic covering of NPs is frequently used to produce NPs for applications involving specific characteristics of the coating material. Due to the lower solubility of their coating properties, they are less applied than the organic polymers for NP delivery in biomedicine. Data on the immune reactions towards inorganic NPs are frequently associated to secondary effects following cell internalization of the particles. NP size and charge play a major role in toxicity, intracellular localization and membrane 
damages that lead to DAMPs release by injured cells and inflammasome activation in the phagocytes. Immune secondary events limit the possibility to find common responses to a certain coating material and restrict the observations to the particles employed in a specific study.

\subsection{Silica}

Silicon dioxide NPs $\left(\mathrm{SiO}_{2} \mathrm{NPs}\right)$ are exploited in a wide range of applications and in particular for biomedical research. Dense or porous silica inorganic coatings are also used to shell different NP cores [66]. $\mathrm{SiO}_{2} \mathrm{NPs}$ have demonstrated low toxicity in vitro suggesting a positive role of silica shell around different core materials, including CdSe/Zn QDs [67-69]. As described earlier, phagocytes such as macrophages and monocytes react more efficiently to micro-size particles than to nano-size particles. Moreover, increased cell damage has been shown for silica micro-particles than for nano-particles [18].

An interesting work by Fruijtier-Polloth and colleagues demonstrated that interaction between amorphous silica nanoparticles and endothelial cells promotes the upregulation of endothelial adhesion molecules expression. This effect enhances the adhesion of monocytes to endothelial cells, a typical early event of inflammatory processes [70]. Moreover, many immunotoxic effects are due to the interference with signaling pathways involved in activation of the immune response or the release of pro-inflammatory cytokines or chemokines [71].

Morishige and colleagues demonstrated that chemical modification of silica NP surfaces can decrease or suppress inflammasome activation and IL-1 $\beta$ release in THP-1 human monocyte/macrophage cell line [25]. However, it is important to note that these inflammatory outcomes have been proved using $1 \mu \mathrm{m}$ particles. Nano-scale silica particles, ranging from 30 to $300 \mathrm{~nm}$, did not induce IL-1 $\beta$ release. These results suggest that inflammatory responses of silica nanoparticles could depend on the combination of several features, including size and surface functionalization.

It is worth mentioning that many indications come from cancer models where the immune system is not in a physiological condition [72]. Further experiments specifically planned would help to better decipher the silica coated NPs impact on the immune system.

\subsection{Gold}

Gold coatings are generally used for the preparation of bimetallic particles, containing a magnetic core as platform for surface functionalization. Some strategies reported in literature describe different core-shell NPs covered with this metal $[73,74]$. Gold-coated nanoparticles (Au-shelled NPs) have been particularly designed for biomedicine, especially for drug delivery [75] and cancer applied hyperthermia treatments [76,77].

There are controversial studies concerning the toxicological effects of engineering gold nanoparticles.

A study by Hashimoto et al. compared the exposure of cultured macrophages RAW264.7 to AuNPs with AgNPs. Although an inflammatory response was observed for both the Au- and Ag-NPs, the harmful cytotoxic effects of AuNPs were smaller than those of the Ag-NPs [78]. However, as described for other kinds of nanomaterials, the interaction between cells and Au nanoparticles could be mediated also by unspecific adsorption of serum proteins onto the gold surface [79]. 


\subsection{Titanium Dioxide $\left(\mathrm{TiO}_{2}\right)$}

Titanium dioxide $\left(\mathrm{TiO}_{2}\right)$ is another coating for inorganic nanoparticles, exploited to increase their cytocompatibility. It was reported that a titanium oxide shell on $\mathrm{Zn}$-based nanoparticles reduces zinc ions release in human lung epithelial cell line [80]. More recent studies demonstrated that core/shell iron oxide/titanium oxide nanoparticles can be used as doxorubicin vector for cancer cells [81]. There are contentious data about $\mathrm{TiO}_{2}$ toxicity. $\mathrm{TiO}_{2}$ particles are considered to be inert and unable to pass undamaged skin. For this reason, they are commonly used for cosmetics or sunscreens preparations as an efficient filter of UV light [82]. In contrast, other studies reported cell toxicity and genotoxicity [83-85]. The discrepancy may be due to different $\mathrm{TiO}_{2}$ particle compositions, different cellular origin, or variation in $\mathrm{TiO}_{2}$ sensitivity between the organisms. $\mathrm{TiO}_{2}$ acts as a modulator of neutrophil degranulation, typically associated with inflammatory process. Actually, it enhances cell surface expression of some granule markers, the secretion of some proteins in the supernatants and metalloproteinases activity in human neutrophils [86].

\section{Discussion and Conclusions}

Nanoparticles designed for biomedical applications in vivo inevitably encounter the human Immune System. Many aspects of nanoparticle-induced modulation of immune responses have been investigated and comprehensive literature on the subject has been published $[9,18]$. In this review we have revised the available literature focusing on the immune aspects specifically due to different surface coatings of NPs, independently of their size, shape and core composition (summary in Table 1). We also excluded the protein functionalization of the surfaces, which could inevitably induce a ligand-receptor driven targeting of the particle or stimulate specific immune pathways on purpose.

It is important to have an overview on this subject; however, it is difficult to draw conclusions or even compare the several ways to shell NPs. The choice of the NP coatings firstly relies on the chemical feature of the employed materials. For example, not all the polymers can be covalently attached or adsorbed onto different metal cores. The use of surfactants may be helpful for solubility improvement, but can completely change the immune response. Experimental approaches that clearly avoid false-positive or false-negative immunotoxicological results of nano-systems are not simple. Secondly, the NPs end goal may limit the application of a specific NP coating. For instance, NPs aimed at clinical applications could require high temperature sterilization processes. These particles cannot be coated with polymers that liquefy at high temperatures. In this case the surface that the immune cells will face would not be the original polymer but either a modified polymer or the core material.

Precise design of NP organic or inorganic coatings to avoid or specifically interact (e.g., coadjuvants) with the Immune System could be done only if the binding sites were clearly known. Polymers differ by chemical groups that present unique composition and structure. The different metallic or metal oxides NPs show diverse reactivity on their surfaces depending on their atomic and crystal structure. So, several features should be considered, like sizes of antibody binding sites $v s$. their potential interaction site on the NP surface. Moreover, the biological conditions and opsonization by diverse molecules in vivo may dramatically alter all these parameters. 
Table 1. Immune response summary of surface coatings.

\begin{tabular}{|c|c|c|c|c|c|}
\hline $\begin{array}{l}\text { Surface } \\
\text { coatings }\end{array}$ & Origin & Immune response & Cell mediator & Applications & References \\
\hline $\begin{array}{l}\text { Polyethylene } \\
\text { glycol (PEG) }\end{array}$ & Synthetic & Complement activation & Phagocytic cells & $\begin{array}{r}\text { Biomedical } \\
\text { applications }\end{array}$ & {$[13,21,26,29,30]$} \\
\hline $\begin{array}{l}\text { Poly(lactic-co } \\
\text { glycolic acid) } \\
\text { (PLGA) }\end{array}$ & Synthetic & Antigen-mediated & $\begin{array}{c}\text { NALP3 } \\
\text { inflammasome } \\
\text { and Caspase } 1 \\
\text { activation } \\
\end{array}$ & $\begin{array}{c}\text { Drug delivery, } \\
\text { vaccine adjuvant }\end{array}$ & [31-35] \\
\hline Dendrimers & Synthetic & $\begin{array}{c}\text { Innate DAMP } \\
\text { receptors-mediated }\end{array}$ & $\begin{array}{l}\text { Dendritic cells } \\
\text { and } \\
\text { macrophages }\end{array}$ & $\begin{array}{l}\text { Anti-infective } \\
\text { ligands }\end{array}$ & {$[36-43]$} \\
\hline Chitosan & Natural & $\begin{array}{c}\text { Innate immune } \\
\text { response }\end{array}$ & $\begin{array}{c}\text { Neutrophils and } \\
\text { macrophages }\end{array}$ & $\begin{array}{l}\text { Coating of drugs, } \\
\text { vaccine adjuvant }\end{array}$ & {$[32,44-49]$} \\
\hline $\begin{array}{l}\text { Sodium } \\
\text { alginate }\end{array}$ & Natural & $\begin{array}{l}\text { Humoral and cellular } \\
\text { response }\end{array}$ & $\begin{array}{l}\text { Neutrophils and } \\
\text { macrophages }\end{array}$ & $\begin{array}{c}\text { Coating for } \\
\text { magnetic and } \\
\text { polymeric NPs, } \\
\text { vaccines } \\
\end{array}$ & {$[33,50,53-55]$} \\
\hline Dextran & Natural & Not well defined & Not well defined & $\begin{array}{l}\text { Anti-platelet } \\
\text { action, metallic } \\
\text { NP coatings } \\
\end{array}$ & {$[27,56-59]$} \\
\hline Starch & Natural & $\begin{array}{l}\text { Low immune and } \\
\text { allergic responses }\end{array}$ & Not well defined & $\begin{array}{c}\text { Skin topical } \\
\text { release, gold or } \\
\text { iron-core } \\
\text { magnetic NPs }\end{array}$ & {$[60-65]$} \\
\hline Silica & Inorganic & $\begin{array}{l}\text { Inflammasome } \\
\text { activation } \\
\text { DAMPs release } \\
\end{array}$ & $\begin{array}{l}\text { Endothelial } \\
\text { cells, } \\
\text { monocytes }\end{array}$ & $\begin{array}{l}\text { Biomedical } \\
\text { research }\end{array}$ & {$[18,25,66-72]$} \\
\hline Gold & Inorganic & $\begin{array}{c}\text { Serum protein } \\
\text { mediated-inflammatory } \\
\text { response }\end{array}$ & Not well defined & $\begin{array}{c}\text { Magnetic } \\
\text { core,cancer } \\
\text { applied } \\
\text { hyperthermia }\end{array}$ & [73-79] \\
\hline $\begin{array}{l}\text { Titanium } \\
\text { dioxide } \\
\left(\mathrm{TiO}_{2}\right) \\
\end{array}$ & Inorganic & $\begin{array}{c}\text { Secretion of proteins, } \\
\text { metalloproteinases } \\
\text { activation } \\
\end{array}$ & Neutrophils & $\begin{array}{l}\text { Vector for cancer } \\
\text { cells, cosmetics }\end{array}$ & [81-85] \\
\hline
\end{tabular}

In view of all this complexity, special attention must also be paid to in vitro and in vivo models for immunology studies. Although currently used in the laboratory, these models provide limited information regarding a potential immune response in human subjects. Immune cell lines are often used as in vitro models. These cell types are proliferating clones that differ from primary immune cells which are extracted from a donor in a physiological state. Primary cells are usually cultured and tested within a couple of weeks. Although the window of time to perform experiments on each donor is shorter than using a cell line, this represents the best in vitro model. Primary white blood cells represent a reliable model to assess the cellular and molecular immune responses following cellular activation pathways by the NPs. The possible release of inflammatory cytokines in the supernatant can also be carefully 
quantified in culture. The weak point of all the in vitro systems is the isolated and artificial environment of the culture. Different sera, media and mutated cell lines of the same origin have produced contradictory results. It is difficult to predict with reasonable certainty what will happen once NPs are administered in vivo. Most of these experiments, however, are necessary to exclude some responses and orientate the researches towards specific outcomes. If high doses of a certain NP do not induce pico-molar doses of inflammatory cytokines in vitro it will be unlikely that the same particle will induce cytokine release in relevant amounts in vivo. On the other hand, a different reaction cannot be excluded in a living organism as a consequence of orchestrated reactions of different cell types.

In vivo experimental models are definitely required to understand systemic immune reactions. The elected model for immunology is the mouse. Selected strains of mice are commercially available and permit the investigation in fast reproducing and easy-to-handle mammals. Their immune system is not exactly like the human one, but the cell types and the main features are very similar. Moreover, modern biotechnology provides genetically modified animals missing genes involved in specific immune reaction. Genetically modified mice for immunity genes provide the most straightforward evidence for potential immune system activation induced by NPs. Model mice with a specific disease offer the opportunity to study the specific immunological events induced by the nano-carrier potentially employed as drug delivery system for the same disease. The latter consideration assumes that specific disease states could present altered immune responses limiting the information collected from experiments of NP release in healthy mice.

In vivo models are mandatory when the targets of delivery are protected organs like the Central Nervous System (CNS). The optimal way to deliver NPs to the CNS would be through the blood circulation. Nonetheless, the presence of the Blood-Brain-Barrier (BBB) increases the level of difficulty for delivering drug carriers. The BBB acts as a rigorously regulated gate for the flow of ions, macromolecules, and nutrients between blood and brain tissue, as well as potential hazards. It combines physical and metabolic barriers to maintain the homeostasis of the CNS [87]. However, reaching the CNS through blood flow is very difficult, although very attractive due to the reduced invasiveness of intravenous administration compared to intracerebroventricular (ICV) or intraparenchymal (IP) injections.

The preparation of NPs for applications in the CNS implies a peculiar design of the surface and specific particle properties. Polymeric NPs or coating with surfactants like polysorbates or PEG have been traditionally used as a strategy to cross the BBB [88-90]. These approaches have been applied with comprehensive chemical design of the nano-vectors and many observations of neural cell responses. Fewer details are available on the immune responses happening in the CNS, once NPs have been introduced in the neural parenchyma.

The CNS resident immune system is represented by microglial cells [91]. These cells are able to sense pathological tissue alteration and perform immunological functions, besides many other emerging functions in maintaining the homeostasis of the healthy CNS.

As already pointed out, PEGylation is one of the most used materials to reduce protein opsonization on the NP surface, limiting the immune system response. It has been observed that uncoated lipid NPs, which accumulate in the brain parenchyma after intravenous injection, cause considerable microglia activation. PEG coating of those lipid NPs with PEG strongly reduce microglial activation [92].

We already mentioned the PEG or PLGA coating of magnetic NPs designed as contrast agents for MRI imaging in the CNS. A novel approach is represented by magnetic core coated with lipid 
modified-PAMAM dendrimers [93]. No indications on immune reactions towards this type of NPs have been presented yet. However, very recent results on G4 amine-PAMAMs and lipid-modified amine-PAMAMs emphasize the importance of the dendrimer modification of the regulation of inflammatory cytokine and chemokine receptors on microglia surface [94]. On the other hand, PAMAM dendrimers alone have already been employed for CNS delivery in vivo demonstrating helpful features for neuro-inflammatory disease models [95].

As described in this review, nanomaterials applied to biomedicine can be designed to avoid or target the immune system. The advent of engineered NPs has highlighted the importance of immunosafety tests to understand the behavior of immune cells in response to these formulations. Unfortunately, nano-sized systems often interfere with the assays usually used by immunologists. Generally, experimental results can be affected by chemical or biological contamination and by optical interference related to the material density. Traditional in vitro tests do not usually contemplate the use of NPs and their possible optical and catalytic interference with the employed reagents and cellular model. Moreover, traces of impurities (solvents, carry-over molecules) and/or endotoxin within a nano-formulation can also induce an inflammatory response, causing cellular- and immunotoxicity [96]. The development of new nanomaterial-aimed techniques and assays will help to validate the already existing results and to unify the results obtained from different methods.

\section{Acknowledgments}

The activity presented in this work has been supported by Fondazione Istituto Italiano di Tecnologia.

\section{Conflict of Interest}

The authors declare no conflict of interest.

\section{References}

1. Labuzek, K.; Gorki, K.; Jaroszek, H.; Jarzabek, K.; Gabryel, B.; Okopien, B. Highly organized nanostructures for brain drug delivery-new hope or just a fad? CNS Neurol Disord Drug Targets 2013, 8, 1271-1285.

2. Wilczewska, A.Z.; Niemirowicz, K.; Markiewicz, K.H.; Car, H. Nanoparticles as drug delivery systems. Pharmacol. Rep. 2012, 64, 1020-1037.

3. Abbas, A.K.; Lichtman, A.H.; Pillai, S. Cellular and Molecular Immunology, 7th ed.; Elsevier: Philadelphia, PA, USA, 2009.

4. Fu, H.; Wang, A.; Mauro, C.; Marelli-Berg, F. T lymphocyte trafficking: Molecules and mechanisms. Front. Biosci. (Landmark Ed) 2013, 18, 422-440.

5. Campbell, K.S.; Hasegawa, J. Natural killer cell biology: An update and future directions. J. Allergy Clin. Immunol. 2013, 132, 536-544.

6. Senovilla, L.; Galluzzi, L.; Zitvogel, L.; Kroemer, G. Immunosurveillance as a regulator of tissue homeostasis. Trends Immunol. 2013, 10, 471-481.

7. Sallusto, F.; Baggiolini, M. Chemokines and leukocyte traffic. Nat. Immunol. 2008, 9, 949-952. 
8. Mithal, D.S.; Banisadr, G.; Miller, R.J. Cxcl12 signaling in the development of the nervous system. J. Neuroimmune Pharmacol. 2012, 7, 820-834.

9. Dobrovolskaia, M.A.; Germolec, D.R.; Weaver, J.L. Evaluation of nanoparticle immunotoxicity. Nat. Nanotechnol. 2009, 4, 411-414.

10. Hubbell, J.A.; Thomas, S.N.; Swartz, M.A. Materials engineering for immunomodulation. Nature 2009, 462, 449-460.

11. Frohlich, E. The role of surface charge in cellular uptake and cytotoxicity of medical nanoparticles. Int. J. Nanomed. 2012, 7, 5577-5591.

12. Zahr, A.S.; Davis, C.A.; Pishko, M.V. Macrophage uptake of core-shell nanoparticles surface modified with poly(ethylene glycol). Langmuir 2006, 22, 8178-8185.

13. Bertrand, N.; Leroux, J.C. The journey of a drug-carrier in the body: An anatomo-physiological perspective. J. Control Release 2012, 161, 152-163.

14. Longmire, M.; Choyke, P.L.; Kobayashi, H. Clearance properties of nano-sized particles and molecules as imaging agents: Considerations and caveats. Nanomedicine (Lond.) 2008, 3, 703-717.

15. Choi, H.S.; Liu, W.; Misra, P.; Tanaka, E.; Zimmer, J.P.; Itty Ipe, B.; Bawendi, M.G.; Frangioni, J.V. Renal clearance of quantum dots. Nat. Biotechnol. 2007, 25, 1165-1170.

16. Lacerda, L.; Herrero, M.A.; Venner, K.; Bianco, A.; Prato, M.; Kostarelos, K. Carbon-nanotube shape and individualization critical for renal excretion. Small 2008, 4, 1130-1132.

17. Ricklin, D.; Hajishengallis, G.; Yang, K.; Lambris, J.D. Complement: A key system for immune surveillance and homeostasis. Nat. Immunol. 2010, 11, 785-797.

18. Dobrovolskaia, M.A.; McNeil, S.E. Handbook of Immunological Properties of Engineered Nanomaterials; World Scientific Publishing: Singapore, 2013.

19. Al-Hanbali, O.; Rutt, K.J.; Sarker, D.K.; Hunter, A.C.; Moghimi, S.M. Concentration dependent structural ordering of poloxamine 908 on polystyrene nanoparticles and their modulatory role on complement consumption. J. Nanosci. Nanotechnol. 2006, 6, 3126-3133.

20. Andersen, A.J.; Hashemi, S.H.; Andresen, T.L.; Hunter, A.C.; Moghimi, S.M. Complement: Alive and kicking nanomedicines. J. Biomed. Nanotechnol. 2009, 5, 364-372.

21. Hamad, I.; Al-Hanbali, O.; Hunter, A.C.; Rutt, K.J.; Andresen, T.L.; Moghimi, S.M. Distinct polymer architecture mediates switching of complement activation pathways at the nanosphere-serum interface: Implications for stealth nanoparticle engineering. ACS Nano 2010, 4, 6629-6638.

22. Hillaireau, H.; Couvreur, P. Nanocarriers' entry into the cell: relevance to drug delivery. Cell Mol. Life Sci. 2009, 17, 2873-2896.

23. Smith, P.J.; Giroud, M.; Wiggins, H.L.; Gower, F.; Thorley, J.A.; Stolpe, B.; Mazzolini, J.; Dyson, R.J.; Rappoport, J.Z. Cellular entry of nanoparticles via serum sensitive clathrin-mediated endocytosis, and plasma membrane permeabilization. Int. J. Nanomed. 2012, 7, 2045-2055.

24. Sun, B.; Wang, X.; Ji, Z.; Li, R.; Xia, T. NLRP3 inflammmasome activation induced by engineered nanomaterials. Small 2013, 9, 1595-1607.

25. Morishige, T.; Yoshioka, Y.; Inakura, H.; Tanabe, A.; Yao, X.; Narimatsu, S.; Monobe, Y.; Imazawa, T.; Tsunoda, S.; Tsutsumi, Y.; et al. The effect of surface modification of amorphous silica particles on NLRP3 inflammasome mediated IL-1 $\beta$ production, ROS production and endosomal rupture. Biomaterials 2010, 31, 6833-6842. 
26. Zhang, F.; Lees, E.; Amin, F.; Rivera Gil, P.; Yang, F.; Mulvaney, P.; Parak, W.J. Polymer-coated nanoparticles: A universal tool for biolabelling experiments. Small 2011, 7, 3113-3127.

27. Tsuchiya, K.; Nitta, N.; Sonoda, A.; Nitta-Seko, A.; Ohta, S.; Takahashi, M.; Murata, K.; Mukaisho, K.; Shiomi, M.; Tabata, Y.; et al. Evaluation of atherosclerotic lesions using dextran- and mannan-dextran-coated uspio: Mri analysis and pathological findings. Int. J. Nanomed. 2012, 7, 2271-2280.

28. Gregory, A.E.; Titball, R.; Williamson, D. Vaccine delivery using nanoparticles. Front. Cell. Infect. Microbiol. 2013, 3, 13.

29. Jevsevar, S.; Kunstelj, M.; Porekar, V.G. Pegylation of therapeutic proteins. Biotechnol. J. 2010, 5, 113-128.

30. Rastogi, R.; Anand, S.; Koul, V. Flexible polymerosomes-An alternative vehicle for topical delivery. Colloids Surf. B Biointerfaces 2009, 72, 161-166.

31. Danhier, F.; Ansorena, E.; Silva, J.M.; Coco, R.; Le Breton, A.; Preat, V. Plga-based nanoparticles: An overview of biomedical applications. J. Control Release 2012, 161, 505-522.

32. Islam, M.A.; Firdous, J.; Choi, Y.J.; Yun, C.H.; Cho, C.S. Design and application of chitosan microspheres as oral and nasal vaccine carriers: An updated review. Int. J. Nanomed. 2012, 7 , 6077-6093.

33. Mata, E.; Igartua, M.; Patarroyo, M.E.; Pedraz, J.L.; Hernandez, R.M. Enhancing immunogenicity to PLGA microparticulate systems by incorporation of alginate and rgd-modified alginate. Eur $J$. Pharm. Sci. 2011, 44, 32-40.

34. Sharp, F.A.; Ruane, D.; Claass, B.; Creagh, E.; Harris, J.; Malyala, P.; Singh, M.; O’Hagan, D.T.; Pétrilli, V.; Tschopp, J.; et al. Uptake of particulate vaccine adjuvants by dendritic cells activates the NALP3 inflammasome. Proc. Natl. Acad. Sci. USA 2009, 106, 870-875.

35. Demento, S.L.; Eisembarth, S.C.; Foellmer, H.G.; Platt, C.; Caplan, M.J.; Saltzman, W.M.; Mellman, I.; Ledizet, M.; Fikrig, E.; Flavell R.A.; Fahmy, T.M. Inflammasome-activating nanoparticles as modular systems for optimizing vaccine efficacy. Vaccine 2009, 27, 3013-3021.

36. Wisher, A.C.; Bronstein, I.; Chechik, V. Thiolated pamam dendrimer-coated cdse/znse nanoparticles as protein transfection agents. Chem. Commun. (Camb.) 2006, 15, 1637-1639.

37. Rouhollah, K.; Pelin, M.; Serap, Y.; Gozde, U.; Ufuk, G. Doxorubicin loading, release, and stability of polyamidoamine dendrimer-coated magnetic nanoparticles. J. Pharm. Sci. 2013, 102, 1825-1835.

38. Pan, B.; Cui, D.; Sheng, Y.; Ozkan, C.; Gao, F.; He, R.; Li, Q.; Xu, P.; Huang, T. Dendrimer-modified magnetic nanoparticles enhance efficiency of gene delivery system. Cancer Res. 2007, 67, 8156-8163.

39. Lamanna, G.; Kueny-Stotz, M.; Mamlouk-Chaouachi, H.; Ghobril, C.; Basly, B.; Bertin, A.; Miladi, I.; Billotey, C.; Pourroy, G.; Begin-Colin, S.; et al. Dendronized iron oxide nanoparticles for multimodal imaging. Biomaterials 2011, 32, 8562-8573.

40. Duncan, R.; Izzo, L. Dendrimer biocompatibility and toxicity. Adv. Drug Deliv. Rev. 2005, 57, 2215-2237. 
41. Witvrouw, M.; Fikkert, V.; Pluymers, W.; Matthews, B.; Mardel, K.; Schols, D.; Raff, J.; Debyser, Z.; De Clercq, E.; Holan, G.; et al. Polyanionic (i.e., polysulfonate) dendrimers can inhibit the replication of human immunodeficiency virus by interfering with both virus adsorption and later steps (reverse transcriptase/integrase) in the virus replicative cycle. Mol. Pharmacol. 2000, 58, 1100-1108.

42. Vannucci, L.; Fiserova, A.; Sadalapure, K.; Lindhorst, T.K.; Kuldova, M.; Rossmann, P.; Horvath, O.; Kren, V.; Krist, P.; Bezouska, K.; et al. Effects of n-acetyl-glucosamine-coated glycodendrimers as biological modulators in the b16f10 melanoma model in vivo. Int. J. Oncol. 2003, 23, 285-296.

43. Shaunak, S.; Thomas, S.; Gianasi, E.; Godwin, A.; Jones, E.; Teo, I.; Mireskandari, K.; Luthert, P.; Duncan, R.; Patterson, S.; et al. Polyvalent dendrimer glucosamine conjugates prevent scar tissue formation. Nat. Biotechnol. 2004, 22, 977-984.

44. Shi, S.F.; Jia, J.F.; Guo, X.K.; Zhao, Y.P.; Chen, D.S.; Guo, Y.Y.; Cheng, T.; Zhang, X.L. Biocompatibility of chitosan-coated iron oxide nanoparticles with osteoblast cells. Int. J. Nanomedicine 2012, 7, 5593-5602.

45. Sun, I.C.; Na, J.H.; Jeong, S.Y.; Kim, D.E.; Kwon, I.C.; Choi, K.; Ahn, C.H.; Kim, K. Biocompatible glycol chitosan-coated gold nanoparticles for tumor-targeting ct imaging. Pharm. Res. 2013, doi:10.1007/s11095-013-1142-0.

46. Mori, T.; Okumura, M.; Matsuura, M.; Ueno, K.; Tokura, S.; Okamoto, Y.; Minami, S.; Fujinaga, T. Effects of chitin and its derivatives on the proliferation and cytokine production of fibroblasts in vitro. Biomaterials 1997, 18, 947-951.

47. Peluso, G.; Petillo, O.; Ranieri, M.; Santin, M.; Ambrosio, L.; Calabro, D.; Avallone, B.; Balsamo, G. Chitosan-mediated stimulation of macrophage function. Biomaterials 1994, 15, 1215-1220.

48. Porporatto, C.; Bianco, I.D.; Correa, S.G. Local and systemic activity of the polysaccharide chitosan at lymphoid tissues after oral administration. J. Leukoc. Biol. 2005, 78, 62-69.

49. Porporatto, C.; Bianco, I.D.; Riera, C.M.; Correa, S.G. Chitosan induces different 1-arginine metabolic pathways in resting and inflammatory macrophages. Biochem. Biophys. Res. Commun. 2003, 304, 266-272.

50. Chen, K.L.; Mylon, S.E.; Elimelech, M. Enhanced aggregation of alginate-coated iron oxide (hematite) nanoparticles in the presence of calcium, strontium, and barium cations. Langmuir 2007, 23, 5920-5928.

51. Demoulins, T.; Bassi, I.; Thomann-Harwood, L.; Jandus, C.; Kaeuper, P.; Simon, H.U.; von Gunten, S.; McCullough, K.C. Alginate-coated chitosan nanogel capacity to modulate the effect of tlr ligands on blood dendritic cells. Nanomedicine 2013, 9, 806-817.

52. Liu, Z.; Lv, D.; Liu, S.; Gong, J.; Wang, D.; Xiong, M.; Chen, X.; Xiang, R.; Tan, X. Alginic acid-coated chitosan nanoparticles loaded with legumain DNA vaccine: Effect against breast cancer in mice. PLoS One 2013, 8, e60190.

53. Yamamoto, Y.; Kurachi, M.; Yamaguchi, K.; Oda, T. Stimulation of multiple cytokine production in mice by alginate oligosaccharides following intraperitoneal administration. Carbohydr. Res. 2007, 342, 1133-1137.

54. Yamamoto, Y.; Kurachi, M.; Yamaguchi, K.; Oda, T. Induction of multiple cytokine secretion from raw264.7 cells by alginate oligosaccharides. Biosci. Biotechnol. Biochem. 2007, 71, 238-241. 
55. Salvador, A.; Igartua, M.; Hernandez, R.M.; Pedraz, J.L. Combination of immune stimulating adjuvants with poly(lactide-co-glycolide) microspheres enhances the immune response of vaccines. Vaccine 2012, 30, 589-596.

56. Yu, M.; Huang, S.; Yu, K.J.; Clyne, A.M. Dextran and polymer polyethylene glycol (peg) coating reduce both 5 and $30 \mathrm{~nm}$ iron oxide nanoparticle cytotoxicity in $2 \mathrm{~d}$ and $3 \mathrm{~d}$ cell culture. Int. J. Mol. Sci. 2012, 13, 5554-5570.

57. Osborne, E.A.; Atkins, T.M.; Gilbert, D.A.; Kauzlarich, S.M.; Liu, K.; Louie, A.Y. Rapid microwave-assisted synthesis of dextran-coated iron oxide nanoparticles for magnetic resonance imaging. Nanotechnology 2012, 23, doi:10.1088/0957-4484/23/21/215602.

58. Jang, H.; Ryoo, S.R.; Kostarelos, K.; Han, S.W.; Min, D.H. The effective nuclear delivery of doxorubicin from dextran-coated gold nanoparticles larger than nuclear pores. Biomaterials 2013, 34, 3503-3510.

59. Lin, Y.S.; Radzi, R.; Morimoto, M.; Saimoto, H.; Okamoto, Y.; Minami, S. Characterization of chitosan-carboxymethyl dextran nanoparticles as a drug carrier and as a stimulator of mouse splenocytes. J. Biomater. Sci. Polym. Ed. 2012, 23, 1401-1420.

60. Yuryev, V.P.; Tomasik, P.; Bertoft, E. Starch: Achievements in Understanding of Structure and Functionality; Nova Science Publishers: Hauppauge, NY, USA, 2007.

61. Lane, M.E. Nanoparticles and the skin—applications and limitations. J. Microencapsul. 2011, 28, 709-716.

62. Hakansson, L.; Hakansson, A.; Morales, O.; Thorelius, L.; Warfving, T. Spherex (degradable starch microspheres) chemo-occlusion-Enhancement of tumor drug concentration and therapeutic efficacy: An overview. Semin. Oncol. 1997, 24, S6-100-S6-109.

63. Pender, D.S.; Vangala, L.M.; Badwaik, V.D.; Willis, C.B.; Aguilar, Z.P.; Sangoi, T.N.; Paripelly, R.; Dakshinamurthy, R. Bactericidal activity of starch-encapsulated gold nanoparticles. Front. Biosci. (Landmark Ed.) 2013, 18, 993-1002.

64. Cole, A.J.; David, A.E.; Wang, J.; Galban, C.J.; Yang, V.C. Magnetic brain tumor targeting and biodistribution of long-circulating peg-modified, cross-linked starch-coated iron oxide nanoparticles. Biomaterials 2011, 32, 6291-6301.

65. Cole, A.J.; David, A.E.; Wang, J.; Galban, C.J.; Hill, H.L.; Yang, V.C. Polyethylene glycol modified, cross-linked starch-coated iron oxide nanoparticles for enhanced magnetic tumor targeting. Biomaterials 2011, 32, 2183-2193.

66. Vivero-Escoto, J.L.; Huxford-Phillips, R.C.; Lin, W. Silica-based nanoprobes for biomedical imaging and theranostic applications. Chem. Soc. Rev. 2012, 41, 2673-2685.

67. Bardi, G.; Malvindi, M.A.; Gherardini, L.; Costa, M.; Pompa, P.P.; Cingolani, R.; Pizzorusso, T. The biocompatibility of amino functionalized $\mathrm{CdSe} / \mathrm{Zn}$ quantum-dot-doped $\mathrm{SiO}_{2}$ nanoparticles with primary neural cells and their gene carrying performance. Biomaterials 2010, 31, 6555-6566.

68. Malvindi, M.A.; Brunetti, V.; Vecchio, G.; Galeone, A.; Cingolani, R.; Pompa, P.P. $\mathrm{SiO}_{2}$ nanoparticles biocompatibility and their potential for gene delivery and silencing. Nanoscale $\mathbf{2 0 1 2}$, 4, 486-495.

69. Asefa, T.; Tao, Z. Biocompatibility of mesoporous silica nanoparticles. Chem. Res. Toxicol. 2012, $25,2265-2284$. 
70. Napierska, D.; Quarck, R.; Thomassen, L.C.; Lison, D.; Martens, J.A.; Delcroix, M.; Nemery, B.; Hoet, P.H. Amorphous silica nanoparticles promote monocyte adhesion to human endothelial cells: Size-dependent effect. Small 2013, 9, 430-438.

71. Fruijtier-Polloth, C. The toxicological mode of action and the safety of synthetic amorphous silica-a nanostructured material. Toxicology 2012, 294, 61-79.

72. Rosenholm, J.M.; Mamaeva, V.; Sahlgren, C.; Linden, M. Nanoparticles in targeted cancer therapy: Mesoporous silica nanoparticles entering preclinical development stage. Nanomedicine (Lond.) 2012, 7, 111-120.

73. Salgueirino-Maceira, V.; Correa-Duarte, M.A.; Farle, M.; Lopez-Quintela, M.A.; Sieradzki, K.; Diaz, R. Synthesis and characterization of large colloidal cobalt particles. Langmuir 2006, 22, 1455-1458.

74. Robinson, I.; Tung le, D.; Maenosono, S.; Walti, C.; Thanh, N.T. Synthesis of core-shell gold coated magnetic nanoparticles and their interaction with thiolated DNA. Nanoscale 2010, 2, 2624-2630.

75. Gangopadhyay, P.; Gallet, S.; Franz, E.; Persoons, A.; Verbiest, T. Novel superparamagnetic core(shell) nanoparticles for magnetic targeted drug delivery and hyperthermia treatment. IEEE Trans. Magn. 2005, 41, 4194-4196.

76. Elsherbini, A.A.; Saber, M.; Aggag, M.; El-Shahawy, A.; Shokier, H.A. Laser and radiofrequency-induced hyperthermia treatment via gold-coated magnetic nanocomposites. Int. J. Nanomedicine 2011, 6, 2155-2165.

77. Elsherbini, A.A.; Saber, M.; Aggag, M.; El-Shahawy, A.; Shokier, H.A. Magnetic nanoparticle-induced hyperthermia treatment under magnetic resonance imaging. Magn. Reson. Imaging 2011, 29, 272-280.

78. Hashimoto, M.; Toshima, H.; Yonezawa, T.; Kawai, K.; Narushima, T.; Kaga, M.; Endo, K. Responses of raw264.7 macrophages to water-dispersible gold and silver nanoparticles stabilized by metal-carbon sigma-bonds. J. Biomed. Mater. Res. A 2013, in press.

79. Chithrani, B.D.; Ghazani, A.A.; Chan, W.C. Determining the size and shape dependence of gold nanoparticle uptake into mammalian cells. Nano Lett. 2006, 6, 662-668.

80. Hsiao, I.L.; Huang, Y.J. Titanium oxide shell coatings decrease the cytotoxicity of zno nanoparticles. Chem. Res. Toxicol. 2011, 24, 303-313.

81. Arora, H.C.; Jensen, M.P.; Yuan, Y.; Wu, A.; Vogt, S.; Paunesku, T.; Woloschak, G.E. Nanocarriers enhance doxorubicin uptake in drug-resistant ovarian cancer cells. Cancer Res. 2012, 72, 769-778.

82. Nohynek, G.J.; Dufour, E.K. Nano-sized cosmetic formulations or solid nanoparticles in sunscreens: A risk to human health? Arch. Toxicol. 2012, 86, 1063-1075.

83. Zhang, R.; Bai, Y.; Zhang, B.; Chen, L.; Yan, B. The potential health risk of titania nanoparticles. J. Hazard Mater. 2012, 211-212, 404-413.

84. Klien, K.; Godnic-Cvar, J. Genotoxicity of metal nanoparticles: Focus on in vivo studies. Arh. Hig. Rada Toksikol. 2012, 63, 133-145.

85. Iavicoli, I.; Leso, V.; Fontana, L.; Bergamaschi, A. Toxicological effects of titanium dioxide nanoparticles: A review of in vitro mammalian studies. Eur. Rev. Med. Pharmacol. Sci. 2011, 15, 481-508. 
86. Babin, K.; Antoine, F.; Goncalves, D.M.; Girard, D. $\mathrm{TiO}_{2}, \mathrm{CeO}_{2}$ and $\mathrm{ZnO}$ nanoparticles and modulation of the degranulation process in human neutrophils. Toxicol. Lett. 2013, 221, 57-63.

87. Abbott, N.J.; Patabendige, A.A.; Dolman, D.E.; Yusof, S.R.; Begley, D.J. Structure and function of the blood-brain barrier. Neurobiol. Dis. 2010, 37, 13-25.

88. Gagliardi, M.; Bardi, G.; Bifone, A. Polymeric nanocarriers for controlled and enhanced delivery of therapeutic agents to the cns. Ther. Deliv. 2012, 3, 875-887.

89. Tosi, G.; Costantino, L.; Ruozi, B.; Forni, F.; Vandelli, M.A. Polymeric nanoparticles for the drug delivery to the central nervous system. Expert Opin. Drug Deliv. 2008, 5, 155-174.

90. Bhaskar, S.; Tian, F.; Stoeger, T.; Kreyling, W.; de la Fuente, J.M.; Grazu, V.; Borm, P.; Estrada, G.; Ntziachristos, V.; Razansky, D. Multifunctional nanocarriers for diagnostics, drug delivery and targeted treatment across blood-brain barrier: Perspectives on tracking and neuroimaging. Part. Fibre Toxicol. 2010, 7, 3.

91. Aguzzi, A.; Barres, B.A.; Bennett, M.L. Microglia: Scapegoat, saboteur, or something else? Science 2013, 339, 156-161.

92. Huang, J.Y.; Lu, Y.M.; Wang, H.; Liu, J.; Liao, M.H.; Hong, L.J.; Tao, R.R.; Ahmed, M.M.; Liu, P.; Liu, S.S.; et al. The effect of lipid nanoparticle pegylation on neuroinflammatory response in mouse brain. Biomaterials 2013, 34, 7960-7970.

93. Boni, A.; Albertazzi, L.; Innocenti, C.; Gemmi, M.; Bifone, A. Water dispersal and functionalization of hydrophobic iron oxide nanoparticles with lipid-modified poly(amidoamine) dendrimers. Langmuir 2013, 29, 10973-10979.

94. Bertero, A.; Boni, A.; Gemmi, M.; Gagliardi, M.; Bifone, A.; Bardi, G. Surface functionalisation regulates polyamidoamine dendrimer toxicity on blood-brain barrier cells and the modulation of key inflammatory receptors on microglia. Nanotoxicology 2014, 2, 158-168.

95. Kannan, S.; Dai, H.; Navath, R.S.; Balakrishnan, B.; Jyoti, A.; Janisse, J.; Romero, R.; Kannan, R.M. Dendrimer-based postnatal therapy for neuroinflammation and cerebral palsy in a rabbit model. Sci. Transl. Med. 2012, 4, doi:10.1126/scitranslmed.3003162.

96. Oostingh, G.J.; Casals, E.; Italiani, P.; Colognato, R.; Stritzinger, R.; Ponti, J.; Pfaller, T.; Kohl, Y.; Ooms, D.; Favilli, F.; et al. Problems and challenges in the development and validation of human cell-based assays to determine nanoparticle-induced immunomodulatory effects. Part. Fibre Toxicol. 2011, 8, doi:10.1186/1743-8977-8-8.

(C) 2014 by the authors; licensee MDPI, Basel, Switzerland. This article is an open access article distributed under the terms and conditions of the Creative Commons Attribution license (http://creativecommons.org/licenses/by/3.0/). 